\title{
Measurements of Proteoglycan and Water Content Distribution in Human Lumbar Intervertebral Discs
}

\author{
James C. latridis, PhD, ${ }^{*}$ Jeffrey J. MacLean, MS, ${ }^{*}$ Mary O'Brien, BA, $\dagger$ \\ and lan A. F. Stokes, PhD*
}

Study Design. Study of regional variations in composition in a sample of 9 mildly to moderately degenerated human intervertebral discs.

Objective. The aim of this study was to obtain proteoglycan distribution in human lumbar discs with high position resolution in the: 1) sagittal, 2) coronal, and 3) axial directions.

Summary of Background Data. Regional variation in disc proteoglycan content has only been reported in coronal sections in a small number of discs and with low spatial resolution in the sagittal direction, and has not been reported in the axial direction.

Methods. Each of 9 human L2-L3 or L3-L4 lumbar discs (age, 53-56 years) were dissected into 36 to 41 specimens using a rectangular cutting die, measured for water content and analyzed for glycosaminoglycan content using the dimethylmethylene blue dye binding assay.

Results. The intervertebral discs were mildly to moderately degenerated. They had glycosaminoglycan content ranging $\sim 40$ to $600 \mu \mathrm{g} / \mathrm{mg}$ dry tissue, with largest values in the nucleus and lowest in the outer anulus. In general, posterior regions had greater glycosaminoglycan content than anterior regions, although values were not as high as in the nucleus. Small variations in glycosaminoglycan content in the axial direction were observed with the largest values in the center, although this variation was small compared with radial variations. Water content results followed similar trends as glycosaminoglycan content with average values ranging from $\sim 66 \%$ to $86 \%$.

Conclusions. A refined map of proteoglycan content is presented with 3 important findings. First, sagittal variations were distinct from coronal variations. Second, the proteoglycan content was not uniform across the nucleus regions. Third, some specimens had localized variations in proteoglycan and water contents suggestive of focal damage and degeneration.

Key words: proteoglycans, aggrecan, glycosaminoglycan content, fixed charge density, intervertebral disc, degeneration. Spine 2007;32:1493-1497
From the "University of Vermont, Burlington, VT; and †Saint Michael's College, Colchester, VT.

Acknowledgment date: August 24, 2006. First revision date: October 26, 2006. Second revision date: November 28, 2006. Acceptance date: November 28, 2006.

Supported by NIH Grant No. R01 AR 049370. Discs were provided by the National Disease Research Interchange.

The manuscript submitted does not contain information about medical device(s)/drug(s).

Federal funds were received in support of this work. No benefits in any form have been or will be received from a commercial party related directly or indirectly to the subject of this manuscript.

Address correspondence and reprint requests to James Iatridis, $\mathrm{PhD}$, University of Vermont, 201 Perkins, 33 Colchester Ave, Burlington, VT 05405; E-mail: james.iatridis@uvm.edu
The distribution of proteoglycan content in the intervertebral disc is functionally important in defining the swelling behaviors, streaming potential, and compressive properties of the tissue. ${ }^{1-4}$ A loss of proteoglycans in the central nucleus pulposus region is a clear sign of early degeneration. ${ }^{5,6}$ The tissue mechanical properties depend on proteoglycan content; therefore, this compositional information is required for mechanical modeling of the disc ${ }^{1,7-10}$ and offers promise for improved understanding of disc function and remodeling in healthy and diseased states.

Previous reports of proteoglycan content distribution in the disc have provided relatively coarse information on the distribution in the sagittal (front to back) direction on a large number of specimens using sulfated glycosaminoglycan (GAG) measurements ${ }^{6}$ or with a higher spatial distribution from a relatively small number of discs using radioactive ion tracers to obtain direct measurements of fixed charge density. ${ }^{11}$ The proteoglycan distributions in the coronal and axial directions have not been reported. In addition to providing an improved understanding of disc physiology in health and disease, a detailed map of the fixed charge density is a missing variable in 3-dimensional finite element analyses of disc behaviors.

The aims of this study were to obtain proteoglycan and water content distributions in human lumbar discs with high position resolution in the sagittal, coronal, and axial directions. We hypothesized that the distribution of proteoglycans in the coronal direction would follow closely that of the sagittal direction having greatest magnitude in the central nucleus and lowest in the outer anulus. We further hypothesized that in the axial direction the proteoglycan content would be greatest in the central region and lower near the upper and lower endplates. Consistent with previous studies, we expected water content distributions to follow similar patterns as proteoglycan content.

\section{Methods}

Human lumbar discs ( $\mathrm{n}=9$ 9; age, 53-56 years; L2-L3 and L3-L4) were obtained on an "as available" basis from the National Disease Research Interchange. To control hydration conditions, motion segments were placed in a saline bath on a testing machine (MTS Systems, Minneapolis, MN) and loaded axially under $500 \mathrm{~N}$ for approximately 3 hours. Motion segments were prepared by cutting through the midtransverse plane of 2 adjacent vertebrae, removing posterior elements and transverse processes, and embedding in polymethylmethacry- 


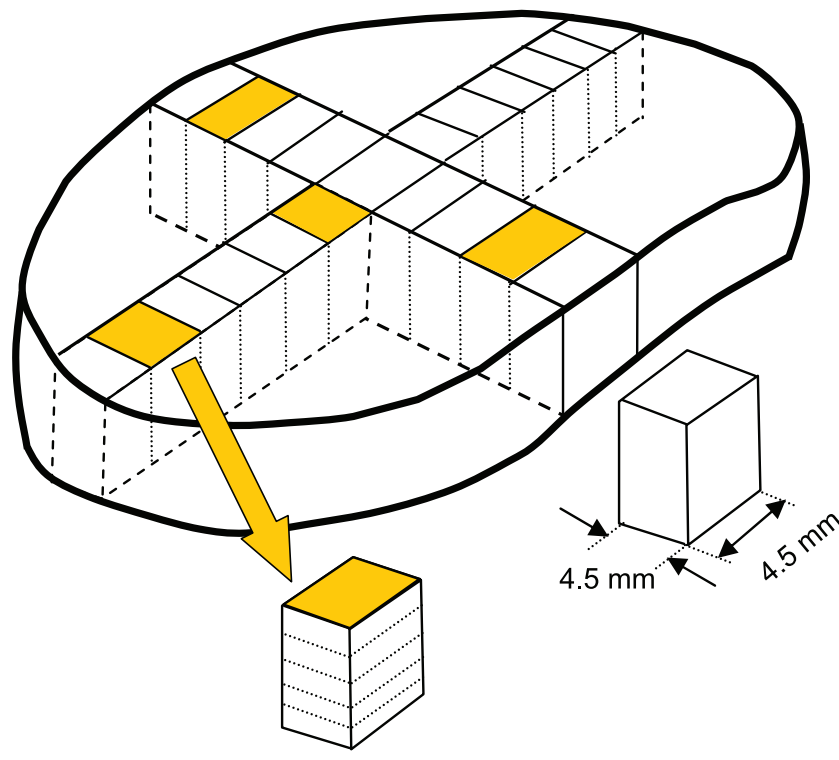

Figure 1. Schematic of disc dissection to obtain proteoglycans and $\mathrm{H}_{2} \mathrm{O}$ distribution in sagittal, coronal, and axial directions. Discs were dissected into 9 to 11 sections (depending on disc size) in the sagittal plane and 11 to 14 sections in the coronal plane. The 4 shaded samples were further dissected into 5 sections as shown to evaluate variations in the axial plane in anterior, posterior, lateral, and nucleus regions.

late. The cut surfaces of the vertebrae were left exposed to allow potential fluid exchange.

Intervertebral discs were then isolated by removing extraneous soft tissue and adjacent vertebrae using a scalpel. Intervertebral discs were then graded by 4 independent observers according to Thompson's 5-point scheme, ${ }^{12}$ modified for transverse sections, and assigned an integer value of the grade closest to the average. Grade 1 corresponds to a completely healthy disc and Grade 5 the most severely degenerated disc.

Each disc was then dissected using a 4.5-mm-wide die-cutter in order to obtain an array of square samples spanning the sagittal direction from anterior to posterior (9-11 sections, depending on disc size) and also spanning the coronal direction from left lateral to central to right lateral locations (11-14 sections, Figure 1). In addition, disc sections from 4 different regions (anterior, posterior, lateral, and nucleus pulposus) were further divided into 5 sections to evaluate the distribution of proteoglycan content in the axial direction from top to bottom of the disc.

A total of 36 to 41 specimens were dissected from each disc with a sum total of 360 specimens analyzed for GAG and water contents. Once dissected, each specimen was placed in preweighed vials, immediately weighed, and then lyophilized to obtain dry weights using a balance with $\pm 0.02 \mathrm{mg}$ repeatability (model AP250D, Ohaus, Pine Brook, NJ). Water content $\left(\% \mathrm{H}_{2} \mathrm{O}\right)$ was calculated as $100 \% \times$ (wet weight-dry weight)/wet weight. Dry tissue was solubilized using proteinase $\mathrm{K}(0.5 \mathrm{mg} / \mathrm{mL}$, Sigma Aldrich, St. Louis, MO) and assayed for GAG content using the dimethylmethylene blue dye binding assay as previously described for disc tissue. ${ }^{6}$ All GAG content measurements were performed in duplicate. GAG content and $\% \mathrm{H}_{2} \mathrm{O}$ values were analyzed as a function of location, and expressed as a percentage of the disc sagittal, coronal, and axial dimensions. Values across all specimens were then averaged by region.

Three separate repeated-measures analyses of variance were performed to evaluate sagittal, coronal, and axial regional differences in GAG and $\% \mathrm{H}_{2} \mathrm{O}$ content with Fisher's PLSD used for a post hoc test. GAG was correlated with $\% \mathrm{H}_{2} \mathrm{O}$ by linear regression analysis. All statistical analyses were performed on Statview (SAS Institute, Cary, NC) using $P<0.05$ as a significance level.

\section{Results}

All intervertebral discs were rated as either Thompson Grade 2 or 3 (2 Grade 2 discs; 7 Grade 3 discs) based on gross morphology, indicating mild to moderate signs of degeneration. The data for 1 disc contained GAG content values (average, minimum, and maximum were: $89.5,18.7$, and $181 \mu \mathrm{g} / \mathrm{mg}$ dry tissue) that were anomalously lower than all other specimens, despite repeated analyses and a similar morphologic grading. This unusual interspecimen variation was attributed to more severe signs of degeneration that was not detectable using the morphologic grading scheme on coronal sections, and so these data were omitted from all analyses.

GAG content was greatest in the nucleus and dropped toward the outer anulus (Figures 2, 3). In general, the posterior regions had slightly greater GAG concentrations than anterior regions, although values were not as
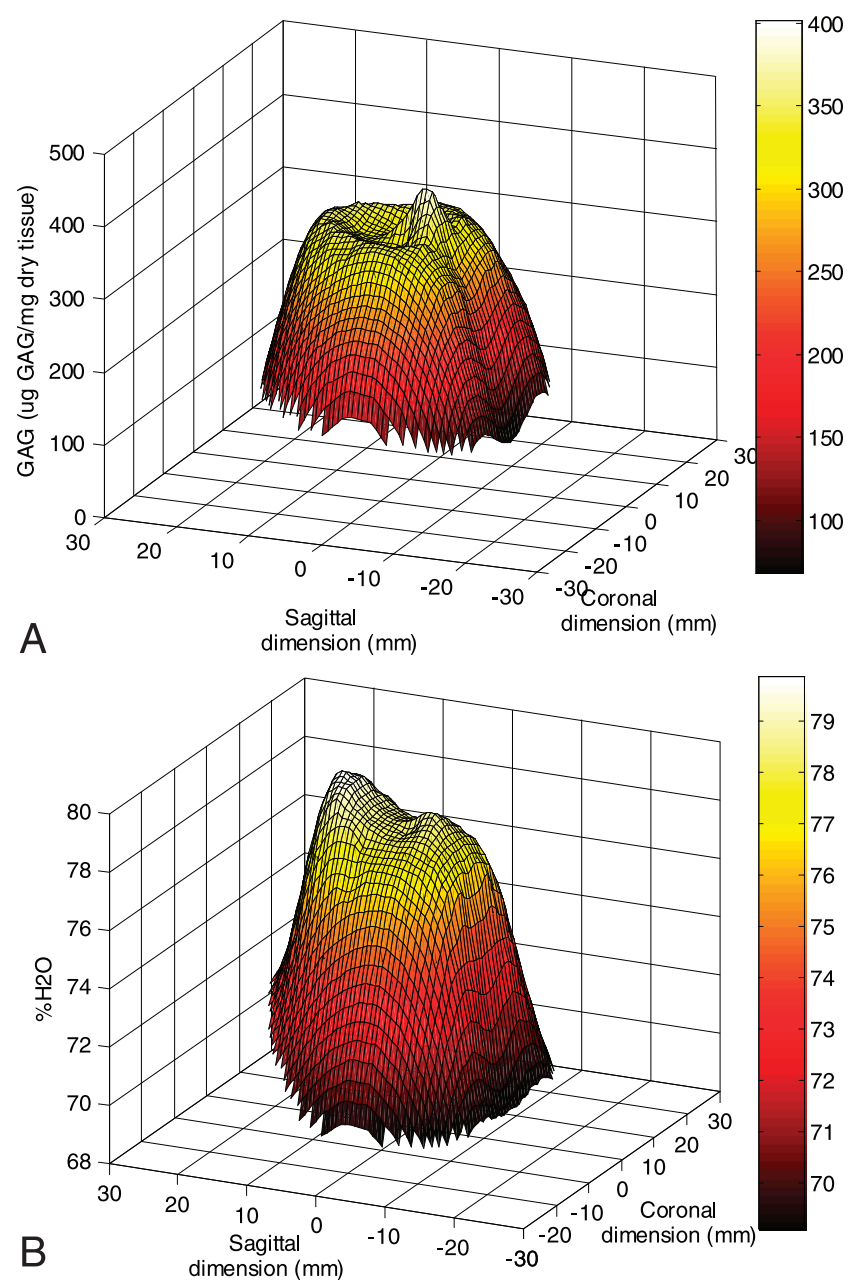

Figure 2. Surface mapping of (A) proteoglycan (GAG) content and (B) water content in sagittal and coronal dimensions. 

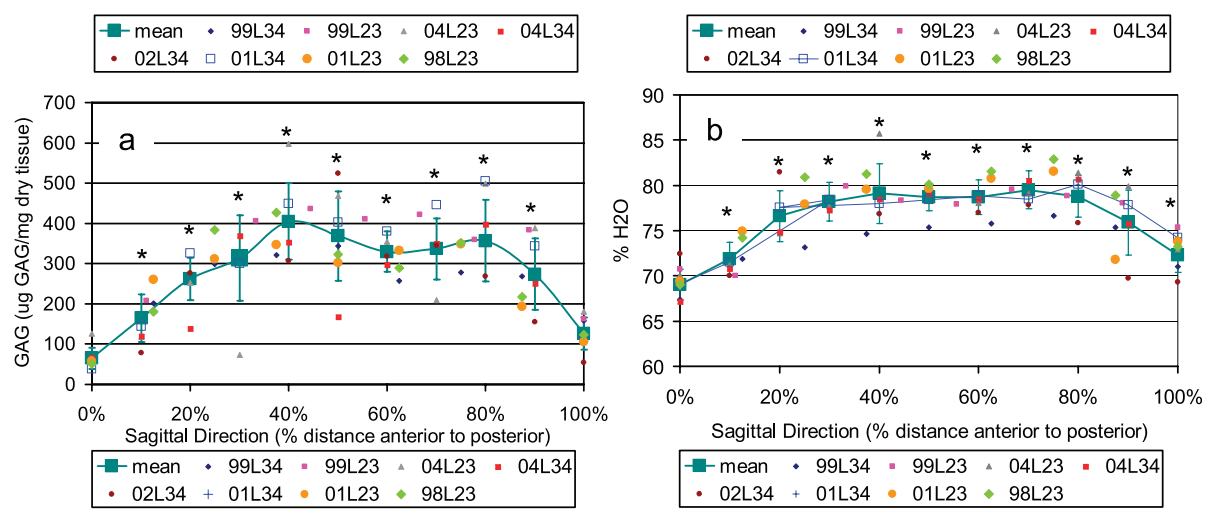

Figure 3. Mean distribution of (a, c, e) GAG and (b, d, f) water content in the sagittal, coronal, and axial directions. For sagittal and coronal distributions, data are presented for each disc along with the pooled mean \pm SD. For axial distributions, data are presented as mean of all 9 specimens in each of 4 regions as well as the pooled mean \pm SD for all discs and all regions. *Significant differences from values at $0 \%$. For axial distributions, $50 \%$ value for GAG was significantly different from $0 \%$ values for the mean location only.
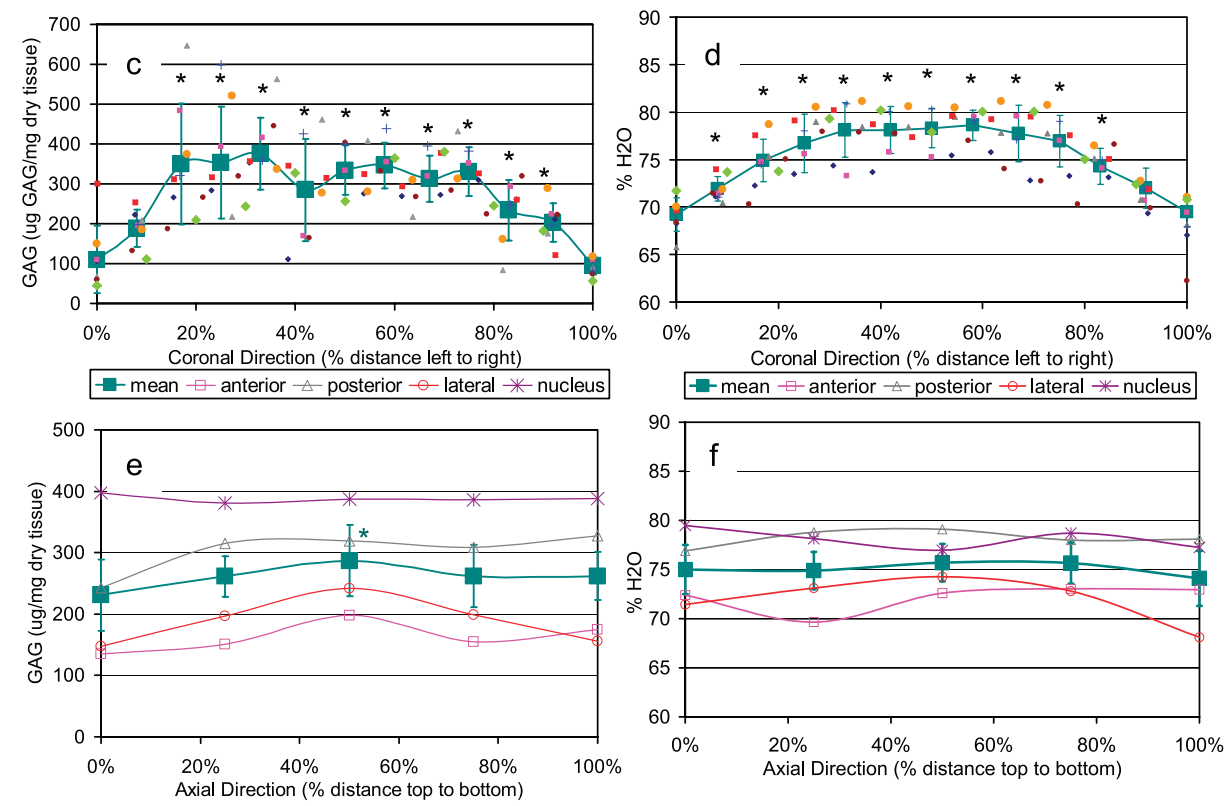

high as in the nucleus. Small variations in GAG in the axial direction were observed with the largest values in the center, although this variation was small compared with radial variations. Water content results generally followed similar trends as GAG although less variable and with average values ranging from $\sim 66 \%$ to $86 \%$. GAG and $\% \mathrm{H}_{2} \mathrm{O}$ had a highly significant but only moderately strong correlation $\left(P<0.001, \mathrm{r}^{2}=0.377, \mathrm{GAG}=\right.$ $-1329+20.9 \times \% \mathrm{H}_{2} \mathrm{O}$ ), indicating the slope was significantly different from zero but that the correlation explained less than $40 \%$ of the experimental variance.

For all specimens, the average GAG value was $250 \pm$ $134 \mu \mathrm{g} \mathrm{GAG} / \mathrm{mg}$ dry tissue weight. Standard deviation (SD) between repeated GAG measurements for each sample was $13.6 \mu \mathrm{g}$ GAG/mg dry tissue. Values for SD in GAG between discs was $73 \mu \mathrm{g}$ GAG/mg wet tissue and was larger within each disc (within disc SD $=111 \mu \mathrm{g}$ GAG/mg dry tissue). For all specimens, the average $\% \mathrm{H}_{2} \mathrm{O}$ value was $75.7 \% \pm 3.96 \%$. Values for $\% \mathrm{H}_{2} \mathrm{O}$ SD calculated between discs was $1.26 \%$ and was also larger within each disc (within disc SD $=7.14 \%$ ). The large variance within each disc for both GAG and $\% \mathrm{H}_{2} \mathrm{O}$ is due predominantly to the strong variations from nucleus to anulus but is also partly due to localized variance within each region of the disc (Figure 3). Spe- cifically, there were 3 discs with patterns that were within the average variance determined in the study for most measurements but also had localized variations more than $1.5 \mathrm{SD}$ away from the average.

\section{Discussion}

These data support previous findings ${ }^{6,13}$ of a high water and proteoglycan content in the central nucleus and low values in the outer anulus but provide a higher position resolution in measurements and also provide new data concerning variations in coronal and axial directions. Several novel and important observations were made. In contrast to our first hypothesis, the coronal variation in water and GAG contents were different from those in the sagittal direction, with values near the posterior nucleus/ transition zone being slightly greater than those in the anterior nucleus/transition zone. Consistent with our second hypothesis, the distribution of GAG and water contents in the axial direction had the largest values in the central region, yet axial direction variations were quite small compared with the large radial variations. Third, average values of GAG and water contents were heterogeneous across the nucleus with a small dip in both compositional parameters in the central nucleus region noted in the mean distribution curves. Fourth, there were 
several disc-specific locations at which GAG measurements had high variation in GAG and $\mathrm{H}_{2} \mathrm{O}$ levels, which may be representative of focal damage and degeneration.

The magnitudes of GAG reported here are very similar to the values reported for Thompson Grade 3 specimens by Antoniou et $a l,{ }^{6}$ although in that paper a wide range in age of specimens was studied to investigate compositional changes with growth, maturation, ageing, and degeneration. Based on measurements of digital photographs of intervertebral disc samples in this study, the nucleus comprised $52 \%$ of the central portion of the sagittal direction and $58 \%$ of the central portion of the coronal direction (Figure 3); however, precise demarcation of nucleus and anulus is difficult in discs with signs of degeneration, and these measurements should be considered only rough guidelines. Interestingly, a small dip in the GAG values in the central nucleus, or intranuclear cleft, was seen in this study, and also reported for Grade 4 specimens, ${ }^{6}$ using fixed charge density values for degenerated specimens, ${ }^{11}$ and with $\mathrm{T} 2$ values on what was considered a normal disc using MRI. ${ }^{13 a}$

The present results demonstrate local variations in proteoglycan content of mild to moderately degenerated discs and are consistent with a previous study on disc composition..$^{5}$ In the present study, localized variance in GAG were not due to measurement error since repeatability between dimethylmethylene blue measurements was small $(<5 \%)$ and wet and dry weight measurements were even smaller $(<1 \%$, minimum sample wet and dry weights were $8.53 \mathrm{mg}$ and $2.42 \mathrm{mg}$, respectively, and balance repeatability was $0.02 \mathrm{mg}$ ). Data from each disc were analyzed separately even when multiple discs were dissected from the same cadaveric spine. This was considered necessary and important because some L2-L3 and L3-L4 discs from the same individual had different grades of degeneration and distinct proteoglycan or water distribution patterns.

Adams and Roughley recently proposed a definition of disc degeneration as an aberrant, cell-mediated response to progressive structural failure. ${ }^{14}$ Morphologic signs of disc degeneration include focal disruptions in the anulus and nucleus. ${ }^{12}$ Focal disruptions in collagen content and collagen crimping have been reported in the human disc. ${ }^{15}$ In the ovine anular lesion model of disc degeneration, focal proteoglycan depletion was measured in areas of nerve and blood vessel in growth into the anulus fibrosus. ${ }^{16}$ Taken together with the literature, we think the localized variations in GAG content found in this study support the contention that mechanisms for disc degeneration involve structural damage and attempts at cell-mediated repair that may result in a localized decrease (or increase) in disc proteoglycan content.

GAG measurements are correlated with or otherwise important for defining functional behaviors of intervertebral discs such as osmotic pressure and streaming potentials. ${ }^{2-4,17-19}$ Nanomechanical measurements of aggrecan interactions also demonstrate the relevance of electrostatic interactions, as described by Poisson-
Boltzmann-based models, in defining how aggrecan molecules contribute to compressive stiffness. ${ }^{20}$ The fixed charge density may be related to GAG concentration using the molecular weight and charge of a chondroitin sulfate disaccharide by: fixed charge density $=[G A G] \times$ $2 / 502.5 \times 10^{3} .^{21,22}$ The most accurate calculations of fixed charge density from GAG must account for the percentage of keratan sulfate to chondroitin sulfate, which is known to increase with aging and degeneration in the disc. ${ }^{5,23}$ Nevertheless, our results are similar to direct measurements of fixed charge density ranging approximately 0.1 to $0.3 \mathrm{mEq} / \mathrm{mg}$ water, ${ }^{13,19}$ and provide a means for obtaining an approximation for fixed charge density measurements in the disc with high degree of spatial resolution.

Overall, this study provides new information characterizing proteoglycan and water content distributions in the 3 primary directions in the intervertebral disc. This information may be helpful for use in 3-dimensional finite element models applying mixture theories while also providing fundamental biochemical data on human discs. Results indicated that sagittal variations in proteoglycan content were distinct from coronal variations, perhaps due to distinct loading patterns in the sagittal and coronal directions. Proteoglycan content was not uniform across the nucleus regions, and there were some localized variations in proteoglycan content that support the contention that mechanisms for disc degeneration involve structural damage and/or attempts at cell-mediated repair that result in localized alterations in disc proteoglycan content.

\section{- Key Points}

- A refined map of proteoglycan content is presented.

- Sagittal variations in proteoglycan content were distinct from coronal variations.

- Proteoglycan content was not uniform across the nucleus regions.

- Some specimens had localized variations in water and proteoglycan content suggestive of focal damage and degeneration.

\section{Acknowledgments}

The authors thank Mr. Mack Gardner-Morse for technical assistance and useful discussions.

\section{References}

1. Iatridis JC, Laible JP, Krag MH. Influence of fixed charge density magnitude and distribution on the intervertebral disc: applications of a poroelastic and chemical electric (PEACE) model. J Biomech Eng 2003;125:12-24.

2. Perie DS, Maclean JJ, Owen JP, et al. Correlating material properties with tissue composition in enzymatically digested bovine annulus fibrosus and nucleus pulposus tissue. Ann Biomed Eng 2006;34:769-77.

3. Urban JP, McMullin JF. Swelling pressure of the lumbar intervertebral discs: influence of age, spinal level, composition, and degeneration. Spine 1988;13: 179-87.

4. Yao H, Justiz MA, Flagler D, et al. Effects of swelling pressure and hydraulic permeability on dynamic compressive behavior of lumbar annulus fibrosus. Ann Biomed Eng 2002;30:1234-41. 
5. Adams P, Eyre DR, Muir H. Biochemical aspects of development and ageing of human lumbar intervertebral discs. Rheumatol Rehabil 1977;16:22-9.

6. Antoniou J, Steffen T, Nelson F, et al. The human lumbar intervertebral disc: evidence for changes in the biosynthesis and denaturation of the extracellular matrix with growth, maturation, ageing, and degeneration. J Clin Invest 1996;98:996-1003.

7. Huyghe JM, Houben GB, Drost MR, et al. An ionised/non-ionised dual porosity model of intervertebral disc tissue. Biomech Model Mechanobiol 2003;2:3-19.

8. Natarajan RN, Williams JR, Andersson GB. Recent advances in analytical modeling of lumbar disc degeneration. Spine 2004;29:2733-41.

9. Schroeder Y, Wilson W, Huyghe JM, et al. Osmoviscoelastic finite element model of the intervertebral disc. Eur Spine J 2006;15(suppl 3):361-71.

10. Wognum S, Huyghe JM, Baaijens FP. Influence of osmotic pressure changes on the opening of existing cracks in 2 intervertebral disc models. Spine 2006;31:1783-8.

11. Urban JPG, Maroudas A. The measurement of fixed charge density in the intervertebral disc. Biochim Biophys Acta 1979;586:166-78.

12. Thompson JP, Pearce RH, Schechter MT, et al. Preliminary evaluation of a scheme for grading the gross morphology of the human intervertebral disc. Spine 1990;15:411-5.

13. Urban JP, McMullin JF. Swelling pressure of the intervertebral disc: influence of proteoglycan and collagen contents. Biorheology 1985;22:145-57.

13a. Haughton V. Imaging intervertebral disc degeneration. J Bone Joint Surg Am 2006;88(suppl):15-20.

14. Adams MA, Roughley PJ. What is intervertebral disc degeneration, and what causes it? Spine 2006;31:2151-61.
15. Gruber HE, Hanley EN Jr. Observations on morphologic changes in the aging and degenerating human disc: secondary collagen alterations. $B M C$ Musculoskelet Disord 2002;3:9.

16. Melrose J, Roberts S, Smith S, et al. Increased nerve and blood vessel ingrowth associated with proteoglycan depletion in an ovine anular lesion model of experimental disc degeneration. Spine 2002;27:1278-85.

17. Best BA, Guilak F, Setton LA, et al. Compressive mechanical properties of the human anulus fibrosus and their relationship to biochemical composition. Spine 1994;19:212-21.

18. Drost MR, Willems P, Snijders H, et al. Confined compression of canine annulus fibrosus under chemical and mechanical loading. J Biomech Eng 1995;117:390-6.

19. Sivan S, Merkher Y, Wachtel E, et al. Correlation of swelling pressure and intrafibrillar water in young and aged human intervertebral discs. J Orthop Res 2006;24:1292-8.

20. Dean D, Han L, Grodzinsky AJ, et al. Compressive nanomechanics of opposing aggrecan macromolecules. J Biomech 2006;39:2555-65.

21. Bashir A, Gray ML, Hartke J, et al. Nondestructive imaging of human cartilage glycosaminoglycan concentration by MRI. Magn Reson Med 1999;41: $857-65$.

22. Burstein D. Calculation of GAG content from a measurement of cartilage T1 in the presence of Gd-DTPA2: Beth Israel Deaconess Medical Center. Available at: http://bidmc.harvard.edu/display.asp?leaf_id $=5597$. Accessed November 28, 2006.

23. Roughley PJ. Biology of intervertebral disc aging and degeneration: involvement of the extracellular matrix. Spine 2004;29:2691-9. 